\title{
Sex Differences in Depressive and Socioemotional Responses to an Inflammatory Challenge: Implications for Sex Differences in Depression
}

\author{
Mona Moieni', Michael R Irwin ${ }^{2}$, Ivana Jevtic', Richard Olmstead ${ }^{2}$, Elizabeth C Breen ${ }^{2}$ and \\ Naomi I Eisenberger*,I \\ 'Department of Psychology, University of California, Los Angeles (UCLA), Los Angeles, CA, USA; ${ }^{2}$ Semel Institute for Neuroscience and Human \\ Behavior, Cousins Center for Psychoneuroimmunology, University of California, Los Angeles (UCLA), Los Angeles, CA, USA
}

\begin{abstract}
Substantial evidence demonstrates that inflammatory processes may underlie depression for a subset of patients, including work showing that healthy subjects exposed to an inflammatory challenge show increases in depressed mood and feelings of social disconnection. However, despite the fact that depression is two times as likely to occur in females than males, the vast majority of this work has been carried out in males. Thus, the objective of this study was to determine whether females (vs males) would show greater increases in proinflammatory cytokines, depressed mood, and social disconnection in response to an inflammatory challenge. One hundred and fifteen healthy participants (69 female) completed this double-blind, placebo-controlled, randomized clinical trial in which participants were randomly assigned to receive either a single infusion of low-dose endotoxin (derived from Escherichia coli; $0.8 \mathrm{ng} / \mathrm{kg}$ of body weight) or placebo (same volume of $0.9 \%$ saline). Interleukin-6 (IL-6), tumor necrosis factor- $\alpha$ (TNF- $\alpha$ ), depressed mood, and feelings of social disconnection were assessed hourly. Results showed that endotoxin (vs placebo) led to increases in proinflammatory cytokines (TNF- $\alpha$, IL-6), depressed mood, and feelings of social disconnection. Females exposed to endotoxin showed greater increases in depressed mood and feelings of social disconnection. Furthermore, increases in TNF- $\alpha$ and IL-6 were correlated with increases in social disconnection for females but not for males. These sex differences in the relationships between inflammatory and socioemotional responses to an inflammatory challenge may be particularly important for understanding why females are two times as likely as males to develop depressive disorders.

Neuropsychopharmacology (20I5) 40, I709-17|6; doi:I0.1038/npp.20I5.17; published online 4 February 20I5
\end{abstract}

\section{Introduction}

Accumulating evidence suggests that inflammation contributes to depression in at least a subset of patients (Raison et al, 2006; Raison and Miller, 2011). Individuals with inflammatory diseases are three to four times more likely to experience depression (Covic et al, 2012; Duivis et al, 2011; Margaretten et al, 2011), patients with major depression have been found to have increased proinflammatory markers (Howren et al, 2009), and an experimental inflammatory challenge (ie, endotoxin-induced inflammation) in an otherwise healthy population can increase depressed mood (Reichenberg et al, 2001). Moreover, recent research suggests that inflammation may contribute to depression, in part, by altering social experience. Thus, endotoxin-induced inflammation can increase feelings of social disconnection

\footnotetext{
* Correspondence: Dr NI Eisenberger, Department of Psychology, University of California, Los Angeles (UCLA), Box 95 I563, 4444 Franz Hall, Los Angeles, CA 90095-1563, USA, Tel: + I 310267 5196, Fax: + I 310206 5895, E-mail: neisenbe@ucla.edu

Received 6 October 2014; revised 8 December 2014; accepted 13 December 2014; accepted article preview online 19 January 2015
}

(Eisenberger et al, 2010) and social anhedonia (Hannestad et al, 2011), and feelings of social disconnection have been shown to mediate the relationship between inflammation and depressed mood (Eisenberger et al, 2010). However, little research has examined sex differences in these effects. In fact, the vast majority of studies investigating the effects of inflammation in relation to depressive symptoms have been conducted in males (reviewed in Schedlowski et al, 2014). Given that females are at a greater risk for depression (Grigoriadis and Robinson, 2007), are more sensitive to social cues (Cyranowski et al, 2000), and show greater inflammatory reactivity (O'Connor et al, 2007), it is important to examine whether females are more susceptible to inflammation-induced socioemotional changes, which may increase risk for depression.

Specifically, females are two times as likely as males to experience depression (Kessler et al, 1993; Weissman et al, 1996; Weissman and Klerman, 1977), and this sex difference is robust, well-documented, and cross-cultural (Cyranowski et al, 2000; Hyde et al, 2008; Kuehner, 2003; Weissman and Klerman, 1977). Interestingly, one factor thought to contribute to the sex difference in depression is females' greater dependence on relationships or affiliative needs 
(Cyranowski et al, 2000; Nolen-Hoeksema, 2001). Indeed, females' greater exposure and reactivity to interpersonal stressors has been shown to contribute to the sex difference in depression (Cyranowski et al, 2000; Hankin and Abramson, 2001; Hyde et al, 2008; Nolen-Hoeksema and Girgus, 1994). Importantly, there are also well-established sex differences in inflammatory processes, such that females are two to nine times more likely to develop autoimmune disorders (Whitacre, 2001), and females show heightened proinflammatory activity (Chapman et al, 2009; O'Connor et al, 2007). However, it is not known whether these sex differences in affiliative preferences or inflammatory activity have a role in inflammatory-related depressive symptoms.

To address these issues, we examined the effect of endotoxin on proinflammatory cytokines, depressed mood, and social psychological experience in a large sample of healthy males and females. We hypothesized that females would show greater increases in proinflammatory cytokines, depressed mood, and feelings of social disconnection.

\section{MATERIALS AND METHODS}

\section{Participants and Procedures}

One hundred and fifteen healthy participants (69 female; mean age: $24.2 \pm 6.6$ years) completed the study. Participants were recruited from UCLA and the greater Los Angeles community using flyers around the UCLA campus, advertisements in campus and local newspapers, and online postings (eg, Craigslist). Interested participants were screened for eligibility using a two-step process, consisting of a structured telephone interview and an in-person screening session. If participants were still eligible, they completed an additional interview at the UCLA Cousins Center for Psychoneuroimmunology to ensure eligibility (see Supplementary Materials and Methods for exclusionary criteria).

The study was conducted between March 2011 and August 2013 (when the intended sample size was reached; see Supplementary Materials) at the UCLA Clinical and Translational Research Center (CTRC) using a randomized, double-blind, placebo-controlled design. Upon arrival to the CTRC, a nurse, who was blind to condition, inserted a catheter with a heparin lock into the dominant forearm (right) for hourly blood draws and one into the nondominant forearm (left) for a continuous saline flush and for drug administration. Ninety minutes after arrival to the CTRC, each participant was randomly assigned to receive either low-dose endotoxin $(0.8 \mathrm{ng} / \mathrm{kg}$ of body weight) or placebo (same volume of $0.9 \%$ saline), which was administered by the nurse as an intravenous bolus. The endotoxin was derived from E. coli (E. coli group O:113: BB-IND 12948 to MRI) and provided by the National Institutes of Health Clinical Center (Suffredini et al, 1999b); previous research has demonstrated the safety of this reference endotoxin across many different samples (Andreasen et al, 2008; Suffredini et al, 1999a).

Throughout the study, vital signs (temperature, pulse, blood pressure, respiration rate; results in Supplementary Materials) and blood draws (to assess cytokine levels) were collected at baseline (T0) and then approximately every hour after injection for the next six hours (T1-T6). Participants also completed hourly measures of sickness symptoms, mood, and feelings of social disconnection (detailed below). Because participants completed a neuroimaging session (reported separately) starting at exactly $2 \mathrm{~h}$ after injection, $T 2$ was assessed before this scanning session at $1 \mathrm{~h}$ and $40 \mathrm{~min}$ after injection and $T 3$ was assessed immediately after the scan at $3 \mathrm{~h}$ and $30 \mathrm{~min}$ after injection; T4-T6 were assessed hourly after T3. For safety reasons, the study physician (MRI) confirmed all the eligibility criteria, was aware of each participant's group assignment, and was on call during each of the experimental sessions, but did not take part in the testing procedures. Participants were discharged from the CTRC following the last blood draw once self-reported physical and psychological symptoms returned to baseline levels, and all participants left the study feeling as well as they did when they started. At the end of the study, participants were thanked, debriefed, and paid for their participation (US\$200 for the day of the study and US\$20 for the prior screening session for a total of US\$220). All subjects provided written consent before participating. All procedures were approved by the UCLA Human Subjects Protection Committee. Because subjects were not asked if their data could be shared, data will be made available upon request (stripped of subjects' identifying information) to be conservative towards protecting subjects.

\section{Behavioral Assessments}

Self-reported sickness symptoms. Physical sickness symptoms (headaches, muscle pain, shivering, nausea, breathing difficulties, and fatigue) were assessed from $T 0$ to $T 6$. Participants rated the extent to which they felt each symptom on a scale from 0 (no symptoms) to 4 (very severe symptoms).

Participants who reported anything other than 'no symptoms' (ie, reporting anything other than ' 0 ') for 3 or more physical sickness symptoms at baseline were removed from all analyses (two participants were removed using these criteria). Additionally, to reduce the likelihood that any between-group differences in social or affective responses were due to severe sickness symptoms, any participant who reported 'very severe' (ie, reporting '4') sickness symptoms for three or more symptoms across all timepoints were removed (two participants were removed using these criteria). For the rest of the participants, scores from each of these items were then dichotomized for each timepoint: any reports of 3 ('severe') or greater for any of the symptoms were scored as a physical symptom response, and 0 otherwise.

Depressed mood. Depressed mood was assessed from T0 to T6 using a short-form version of the Profile of Mood States (Baker et al, 2002; McNair et al, 1971). Participants rated the extent to which they felt: 'unhappy', 'sad', blue', 'hopeless', 'discouraged', 'miserable', 'helpless', and 'worthless' on a scale from 0 (not at all) to 4 (extremely). Measures of depressed mood were calculated by summing scores from each of these items at each timepoint. The reliability of the scale (assessed at the time of peak response) was high $(\alpha=0.83)$.

Feelings of social disconnection. Feelings of social disconnection were also assessed from T0 to T6. Participants rated items such as: 'I feel lonely', 'I feel disconnected 
from others', and 'I feel isolated from others' on a scale from 1 (not at all) to 5 (very much so). The questionnaire included 12 items and was created using items from a social disconnection scale (Eisenberger et al, 2010), a loneliness scale (Joiner et al, 2002), and adding two additional items: 'I feel lonely' and 'I feel liked' (Russell, 1996), and scores were averaged at each timepoint to create a measure of selfreported social disconnection. The reliability of the scale (assessed at the time of peak response) was high $(\alpha=0.86)$.

\section{Plasma Levels of Cytokines: Information about Assays}

Whole blood samples were collected in prechilled EDTA tubes. After collection, the samples were centrifuged at $4{ }^{\circ} \mathrm{C}$, plasma was harvested into multiple aliquots, and then stored in a $-70^{\circ} \mathrm{C}$ freezer until the completion of the study.

Using a Bio-Plex 200 (Luminex) Instrument, Bio-Plex software v.4.1, and a five-parameter logistic curve fit, plasma levels of IL- 6 and TNF- $\alpha$ were quantified by means of high sensitivity bead-based multiplex immunoassays (Performance High Sensitivity Human Cytokine; R\&D Systems, Minneapolis, MN). This R\&D Systems multiplex assay has been shown to have excellent intra- and interassay reproducibility for these two analytes in a recent temporal stability study of circulating cytokine levels (Epstein et al, 2013) and very strong correlations $(r \geqslant 0.94)$ across a wide range of concentrations with high-sensitivity ELISA Kits from the same manufacturer (Breen et al, 2014). All multiplex assays were performed on plasma samples diluted twofold according to the manufacturer's protocol, and all calculated concentrations generated by the BioPlex Manager software were included in data analyses. Owing to the strength of the parent study design (Eisenberger et al, 2009, 2010), which used repeated measures of cytokine values over seven timepoints for each subject, each timepoint was evaluated in a single determination. Every subject demonstrated the expected profile of change of cytokine concentrations over time, based on these previous studies (Eisenberger et al, 2009, 2010).

Paired samples from each subject (baseline and all subsequent timepoints) were assayed on the same 96-well plate; multiplex assays were chosen for the analyses because of the large dynamic range necessary to evaluate both low physiologic (baseline) and very high (postendotoxin) cytokine concentrations in the same assay. The ranges of detection for IL-6 and TNF- $\alpha$ were $0.2-3800$ and $0.8-$ $3100 \mathrm{pg} / \mathrm{ml}$, respectively, and no samples exceeded the upper limit of detection for either analyte. The mean intraassay CV\% of the standards was $<8 \%$ for IL- 6 and TNF- $\alpha$; the interassay $\mathrm{CV} \%$ of an internal laboratory quality control sample was $<13 \%$ for both analytes.

\section{Statistical Analyses}

First, to establish between-group differences in the effect of endotoxin $v s$ placebo on cytokines, depression, and social disconnection across time, we used a standard statistical program (SPSS 22.0) to conduct an omnibus (from $T 1$ to T6) repeated-measures analyses of covariance (ANCOVA) examining condition by time interactions controlling for baseline values. ANCOVA was chosen in light of evidence suggesting that this method ( $v s$ using repeated measures to detect changes from baseline) increases statistical power in randomized studies and adjusts for baseline imbalances (Van Breukelen, 2006; Winkens et al, 2007). Next, to examine where these differences occurred, we conducted ANCOVAs examining condition effects at specific timepoints (eg, T1) controlling for baseline values. Additionally, to examine potential sex differences in the effects of endotoxin, we conducted ANCOVAs examining condition by sex interactions at each timepoint controlling for baseline.

Because the cytokine values were not normally distributed at any timepoint, values were log-transformed, and because of known effects of body mass index (BMI) on cytokines, we controlled for BMI in all cytokine analyses. Additionally, subjects whose scores were over 3 SDs on the dependent variables of interest were removed from the respective analyses to improve the robustness of the results to replication, improve accuracy, and reduce errors (Osborne and Overbay, 2004).

Finally, to examine differences in self-reported sickness symptoms, a dichotomous outcome, we used a nonparametric Fisher's exact test to examine differences in sickness symptoms by condition (endotoxin $v s$ placebo) at each timepoint.

\section{RESULTS}

\section{Characteristics of the Sample}

Participants who were outliers $( \pm 3$ SD) on the Beck Depression Inventory (Beck et al, 1988) were removed from all analyses (two participants were removed using these criteria). Additionally, as mentioned above, four participants were removed for being out of range on sickness symptoms. Of the remaining 109 participants (65 female; mean age: $24.1 \pm 6.3$ years), 51 were randomized into the placebo condition and 58 were randomized into the endotoxin condition. The groups were not significantly different $(p$ 's $>0.1)$ on the demographic variables presented in Table 1 . There were no sex differences ( $p$ 's $>0.1$ ) on these variables except for age, where males were older than females $(t(107)=2.49, p<0.05)$. Thus, we ran additional analyses controlling for age for all relevant analyses below looking at sex differences; the results were unchanged when controlling for age.

\section{Physiological Responses to Endotoxin}

Cytokines. As expected, an omnibus test revealed that endotoxin ( $v s$ placebo) led to significant increases in IL-6 (Figure 1a; $\mathrm{F}(5,490)=132.71, p<0.001$ ) and TNF- $\alpha$ levels (Figure $1 b ; F(5,500)=187.47, p<0.001$ ) over time. Condition effects of endotoxin $v s$ placebo at each timepoint controlling for baseline were significant from $T 1$ to $T 6$ for both IL- 6 and TNF- $\alpha(p$ 's $<0.001)$. There were no sex differences in the effect of endotoxin on IL-6 or TNF- $\alpha$ levels; that is, there were no significant sex by condition interactions at any timepoint controlling for baseline for either IL-6 or TNF- $\alpha$ ( $p$ 's $>0.3)$.

Self-reported sickness symptoms. Endotoxin (vs placebo) participants reported significantly greater sickness symptoms at $T 2$ (Fisher's exact $p<0.001$ ), but not at any other timepoints (Fisher's exact $p$ 's $>0.2$ ). At T2, $28 \%$ of 
Table I Characteristics of the Sample

\begin{tabular}{|c|c|c|c|c|}
\hline & $\begin{array}{l}\text { Placebo males } \\
\quad(n=22)\end{array}$ & $\begin{array}{l}\text { Placebo females } \\
\quad(n=29)\end{array}$ & $\begin{array}{l}\text { Endotoxin males } \\
\quad(n=22)\end{array}$ & $\begin{array}{l}\text { Endotoxin females } \\
\qquad(n=36)\end{array}$ \\
\hline Mean age (SD) & $26.3(8.0)$ & $21.4(2.7)$ & $25.5(5.9)$ & $24.2(6.7)$ \\
\hline Mean body mass index (SD) & $23.8(2.6)$ & $23.2(2.8)$ & $24.6(2.5)$ & $24.2(3.2)$ \\
\hline \multicolumn{5}{|l|}{ Race (\%) } \\
\hline African American & 0 & 6.9 & 4.5 & 2.8 \\
\hline Latino & 0 & 27.6 & 27.3 & 30.6 \\
\hline Other & 4.5 & 6.9 & 4.5 & 11.1 \\
\hline Alcohol use in $24 \mathrm{~h}$ before experimental session (\% who said 'yes') & 0 & 0 & 0 & $2.8(n=1)$ \\
\hline Smoking in $24 \mathrm{~h}$ before experimental session (\% who said 'yes') & 0 & 0 & 0 & 0 \\
\hline
\end{tabular}
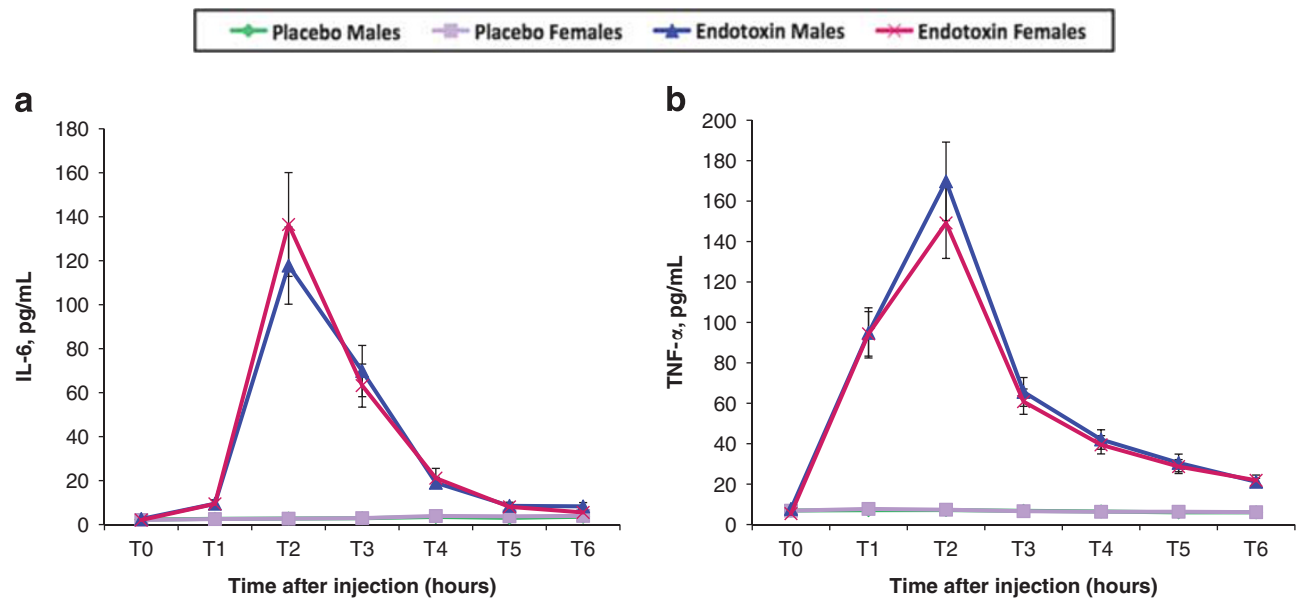

Figure I Changes in cytokines. Changes over time in the endotoxin and placebo groups (split by sex) in plasma levels of (a) interleukin-6 (IL-6) and (b) tumor necrosis factor- $\alpha$ (TNF- $\alpha$ ) (raw values that have not been log-transformed are shown). Cytokines were assessed at baseline (T0) and then approximately every hour after injection for the next $6 \mathrm{~h}(T I-T 6)$. Because participants completed a neuroimaging session (reported separately) starting at exactly $2 \mathrm{~h}$ after injection, $T 2$ was assessed before this scanning session at I $\mathrm{h}$ and $40 \mathrm{~min}$ after injection, and $T 3$ was assessed immediately after the scan at $3 \mathrm{~h}$ and $30 \mathrm{~min}$ after injection; T4-T6 were assessed hourly after T3. Error bars depict the standard error of the mean.

endotoxin participants reported at least one 'severe' or 'very severe' symptom, compared with $0 \%$ of placebo participants. There were no sex differences in sickness symptoms at any timepoint (Fisher's exact $p$ 's $>0.2$ )

\section{Social and Affective Responses to Endotoxin}

Depressed mood. Consistent with prior work (Eisenberger et al, 2010; Reichenberg et al, 2001), an omnibus test revealed that endotoxin ( $v s$ placebo) led to significantly greater increases in depressed mood over time, adjusted for baseline (Figure 2a; $\mathrm{F}(5,460)=7.64, p<0.001)$. Analyses at each timepoint revealed that endotoxin ( $v s$ placebo) led to significant increases in depressed mood at $T 2(\mathrm{~F}(1,101)=11.09$, $p<0.01)$. These effects did not change when controlling for sickness symptoms at $T 2(\mathrm{~F}(1,99)=4.08, p<0.05)$.
Additionally, there were significant condition (endotoxin $v s$ placebo) by sex interactions in depressed mood at $T 1$ $(\mathrm{F}(1,99)=5.12, p<0.05)$ and $T 2(\mathrm{~F}(1,99)=5.91, p<0.05)$, controlling for baseline. Females displayed significantly greater increases in depressed mood than males in response to endotoxin at $T 2(\mathrm{~F}(1,51)=4.80, p<0.05)$ and marginally at $T 1 \quad(F(1,53)=3.68, p=0.06)$, controlling for baseline values; however, among those who received placebo, there were no sex differences ( $p$ 's $>0.1$ ). These sex differences did not change after controlling for sickness symptoms at $T 1$ $(\mathrm{F}(1,99)=5.12, p<0.05)$ or $\mathrm{T} 2(F(1,97)=5.44), p<0.05)$.

Feelings of social disconnection. As shown previously (Eisenberger et al, 2010), endotoxin ( $v s$ placebo) led to significantly greater increases in feelings of social disconnection 
$\leadsto$ Placebo Males - Placebo Females $\pitchfork$ Endotoxin Males $\quad$ Endotoxin Females

a

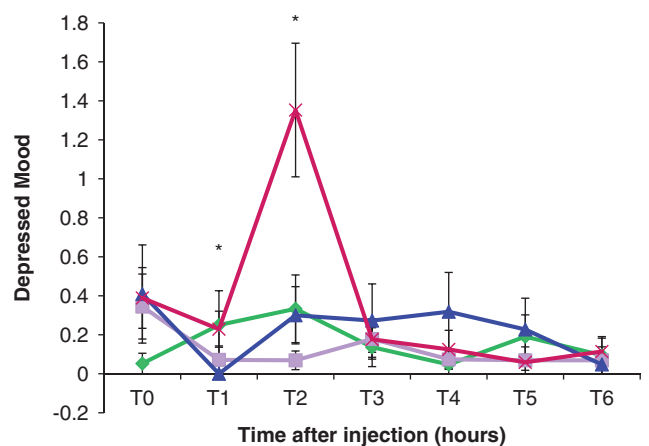

b

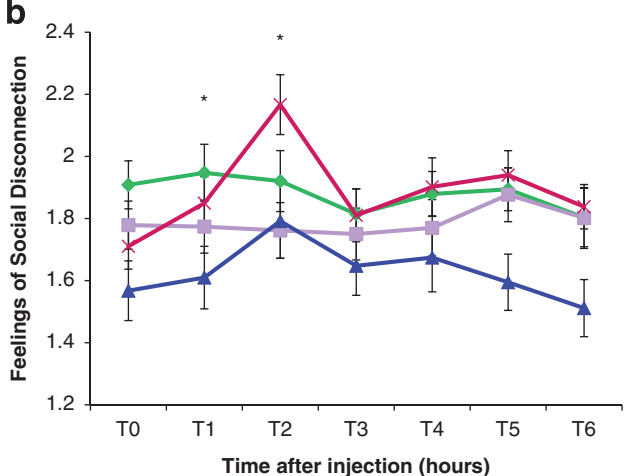

Figure 2 Changes in socioemotional responses. Changes over time in the endotoxin and placebo groups (split by sex) in (a) depressed mood and (b) feelings of social disconnection. Similar to the assessment of cytokines, socioemotional responses were assessed at baseline (T0) and then approximately

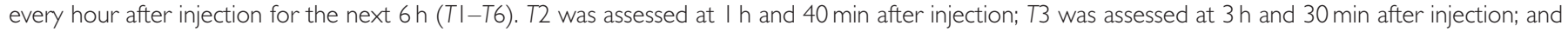

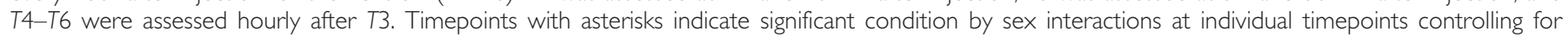
baseline values. Error bars depict the standard error of the mean.

over time, adjusted for baseline (Figure $2 \mathrm{~b} ; \mathrm{F}(5,505)=4.35$, $p<0.01)$. Analyses at each timepoint revealed that endotoxin ( $v s$ placebo) led to significant increases in social disconnection at $T 2(\mathrm{~F}(1,106)=13.04, p<0.001)$ and $T 4$ $(\mathrm{F}(1,103)=4.01, p<0.05)$. These effects remained when controlling for sickness symptoms at $T 2(\mathrm{~F}(1,104)=5.19$, $p<0.05)$ and T4 $(\mathrm{F}(1,102)=3.26, p=0.07)$.

Importantly, there were also significant condition (endotoxin $v s$ placebo) by sex interactions on changes in felt social disconnection at $T 1(\mathrm{~F}(1,103)=4.77, p<0.05)$ and $T 2$ $(\mathrm{F}(1,104)=6.02, p<0.05)$ and a marginal condition by sex interaction at $T 5(\mathrm{~F}(1,104)=3.9, p=0.05)$ controlling for baseline values. Females showed greater increases in feelings of social disconnection than males in response to endotoxin at $T 1(\mathrm{~F}(1,55)=5.54, p<0.05), T 2(\mathrm{~F}(1,55)=$ $7.44, p<0.01)$, and $T 5(\mathrm{~F}(1,55)=14.83, p<0.01)$. There were no significant sex differences within the placebo group ( $p$ 's $>0.4)$. These effects remained significant when controlling for sickness symptoms at $T 1(\mathrm{~F}(1,103)=4.77, p<0.05)$, T2 $(\mathrm{F}(1,102)=5.41, p<0.05)$, and T5 $(\mathrm{F}(1,102)=4.00$, $p<0.05)$.

\section{Relationships between Cytokines and Affective and Social Responses}

To better understand the sex differences in the inflammatory-induced changes in affective and social responses, we also examined whether there were sex differences in the relationships between changes in cytokines and changes in affective and social responses. To do this, we looked at correlations between changes from $T 0$ to $T 2$ in cytokines (ie, IL-6, TNF- $\alpha$ ) and affective and social changes (ie, depressed mood, social disconnection) in the whole endotoxin sample as well as for males and females separately. We focused on changes from $T 0$ to $T 2$ for two reasons: (1) $T 2$ was the peak of cytokine response for both IL- 6 and TNF- $\alpha$, and (2) T2 was the only timepoint during which endotoxin ( $v s$ placebo) subjects reported both significantly greater depressed mood and feelings of social disconnection. All correlational analyses controlled for BMI.
For the full sample, there were significant correlations between changes in IL- 6 and social disconnection $(r=0.27$, $p<0.05)$, as well as between changes in TNF- $\alpha$ and social disconnection $(r=0.37, p<0.01)$. The correlation between changes in IL- 6 and depressed mood was marginal $(r=0.25$, $p=0.08)$. Finally, the correlation between changes in TNF- $\alpha$ and depressed mood was significant $(r=0.30, p<0.05)$

When examining males and females separately, the correlation between changes in IL- 6 and social disconnection was significant for females (Figure 3a; $r=0.40, p<0.05$ ) but not for males (Figure $3 b ; r=-0.04, p=0.88$ ). Similarly, the correlation between changes in TNF- $\alpha$ and social disconnection was significant for females (Figure $3 \mathrm{c}$; $r=0.46, p<0.01$ ) but not for males (Figure $3 \mathrm{~d} ; r=0.09$, $p=0.69)$. The correlations between changes in IL-6 or changes in TNF- $\alpha$ and depressed mood were not significant for males or females ( $p$ 's $>0.1)$.

\section{DISCUSSION}

Given mounting evidence showing that females are at a greater risk for depression, are more sensitive to the social environment, and show greater inflammatory activity, it is critical to examine sex differences in the effect of inflammation on social experience that may increase risk for depression. Here, we demonstrated that in response to an inflammatory challenge, females showed greater increases in depressed mood and social disconnection. Furthermore, increases in proinflammatory cytokines (ie, TNF- $\alpha$, IL-6) corresponded directly with increases in felt social disconnection for females but not for males.

Investigating sex differences has been largely overlooked in this literature, where the majority of studies have been conducted in male-only samples (reviewed in Schedlowski et al, 2014). The sex differences found in this study in the relationships between inflammatory and socioemotional responses may be particularly important as they may have implications for understanding why females are two times as likely as males to develop depressive disorders 

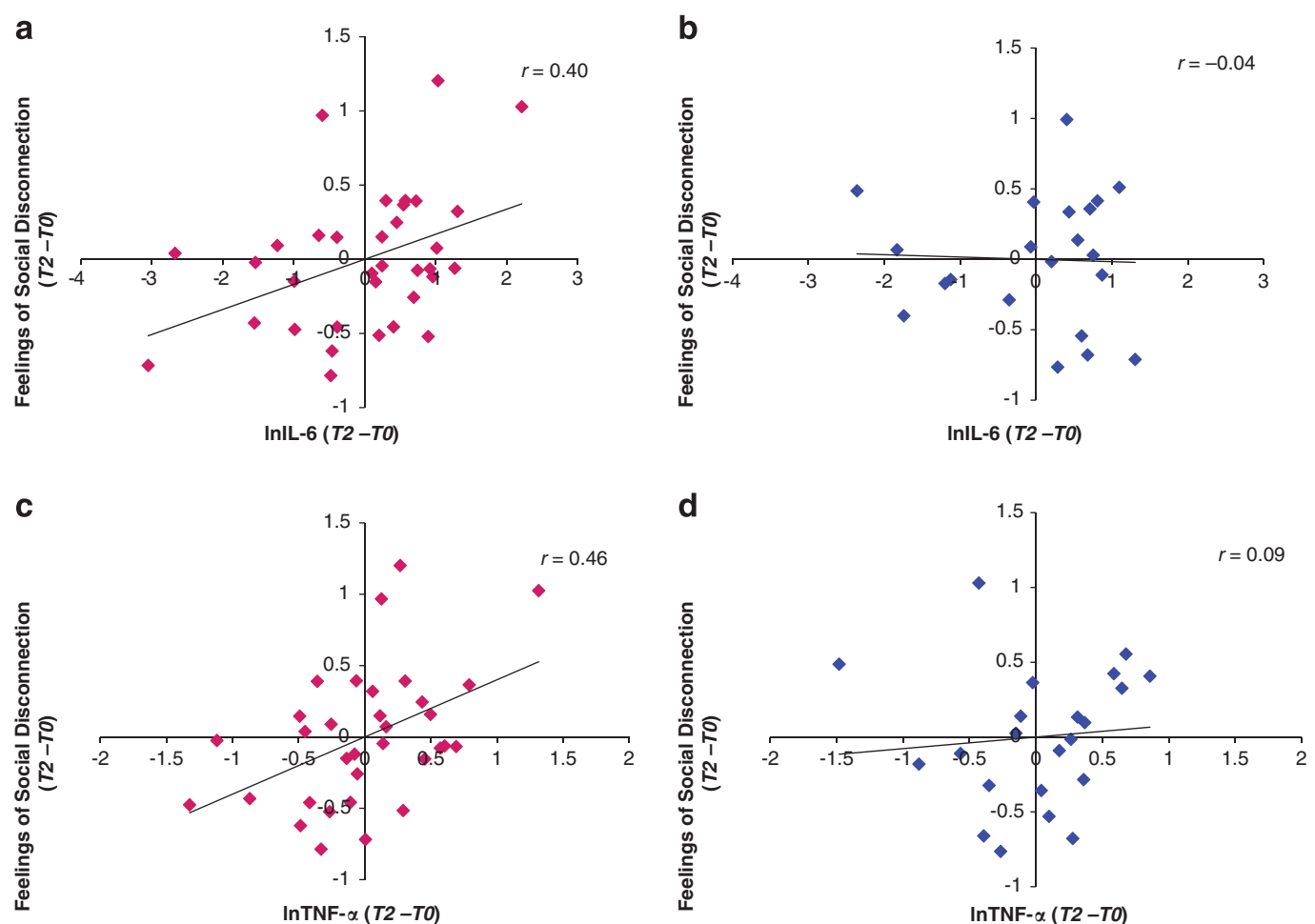

Figure 3 Correlations between social disconnection and cytokines. Correlations between changes (T2-T0) in feelings of social disconnection and changes in interleukin-6 (IL-6) (T2-TO) reported separately for (a) females and (b) males, and correlations between changes (T2-T0) in feelings of social disconnection and changes in tumor necrosis factor- $\alpha$ (TNF- $\alpha$ ) for (c) females and (d) males. All values reported here and all analyses controlled for body mass index (BMI).

(Grigoriadis and Robinson, 2007; Weissman et al, 1996). For example, in the present study, females ( $v s$ males) showed greater increases in depressed mood in response to endotoxin, indicating that females may be more sensitive to the affective consequences of inflammation, which given the potential role of inflammation in the pathogenesis of depression (Raison and Miller, 2011), may contribute to the increased likelihood for females to develop depressive disorders.

Furthermore, females also showed greater increases in feelings of social disconnection in response to endotoxin compared with males. Importantly, feelings of social disconnection contribute to the onset and perpetuation of depression (Heinrich and Gullone, 2006), and it has been argued that females' greater dependence on social relationships may contribute to the sex differences in depression (Cyranowski et al, 2000). The present findings indicate that females experience more pronounced changes in feelings of social disconnection in response to increases in inflammation. Thus, this increased sensitivity to changes in social experience may also have a role in the sex differences in rates of depression.

Finally, there were no sex differences in proinflammatory responses. Although some work has shown that females show heightened proinflammatory activity (Chapman et al, 2009; O'Connor et al, 2007), the literature is equivocal. Indeed, some have suggested that sex differences in inflammatory markers may be due to differences in adiposity (Cartier et al, 2009). Furthermore, because the majority of human studies using endotoxin did not include females (reviewed in Schedlowski et al, 2014), we looked to another experimental context (ie, psychological stress) that elicits inflammatory responses, where findings regarding sex differences in cytokine responses are also mixed (Steptoe et al, 2007). For example, one study found that females showed greater IL- 6 responses but smaller TNF- $\alpha$ to stress compared with males (Steptoe et al, 2002). Another study found that while there were no sex differences in the magnitude of the increase in cytokine production in response to social stress, there were sex differences in the recovery period (Prather et al, 2009).

Additionally, in the context of the relationship between inflammation and depression, findings about sex differences are also mixed. Some correlational work has found that the relationship between inflammatory markers and symptoms of depression or depressed mood is stronger in males than females (Gimeno et al, 2009; Penninx et al, 2003). Conversely, in the context of clinical studies that follow patients using the cytokine interferon- $\alpha$ (IFN- $\alpha$ ), some work has found that females who receive IFN- $\alpha$ are at greater risk of developing depression than males (Gohier et al, 2003; Koskinas et al, 2003). Still, others have reported no sex differences in either correlational relationships between inflammation and depression (Bremmer et al, 2008) or in IFN- $\alpha$-induced depression (Bonaccorso et al, 2002). These mixed findings suggest that further studies are needed to better understand sex differences in this literature.

However, although there were no sex differences in proinflammatory responses in the present study, the relationship between changes in proinflammatory cytokines and changes in social disconnection was significant for females 
but not for males. This finding indicates that females may be particularly sensitive to the social psychological changes that accompany increases in inflammation. This increased social sensitivity to changes in cytokines may further contribute to the increased likelihood for females to develop depressive disorders.

In addition to providing insight about sex differences in depressive disorders, this study may ultimately have other clinical implications if corroborated by future studies. Namely, because these data suggest that women are more sensitive to the socioemotional consequences of heightened inflammation, females diagnosed with disorders marked by chronic inflammation (eg, arthritis, multiple sclerosis) may be particularly vulnerable to developing depressive disorders. Although the literature is mixed, there is some evidence to support this notion. For example, one study found that females with arthritis had 33\% higher odds of major depression than males (Fuller-Thomson and Shaked, 2009), and other work has found that females with multiple sclerosis have a higher prevalence of depression than males (Patten et al, , 2000, 2003). These data, in combination with the results from this study, would suggest that clinicians may want to more carefully monitor females with disorders characterized by chronic inflammation.

A few limitations should be considered. First, while one of the study's strengths was looking at measures of social psychological experience, we did not measure social behavior directly. Future work should examine the effect of experimental inflammation on behavior in social situations to determine whether the sex differences present in this study extend to sex differences in social behavior. Furthermore, while this study can aid our understanding of the role of inflammation in depression and sex differences in rates of depression, this study was conducted using a healthy, young sample. Thus, additional work is needed to better understand these processes in both older and clinically depressed samples.

In conclusion, these findings make an important contribution to the literature by providing evidence for sex differences in alterations in mood and social psychological experience from an experimental inflammatory challenge. In response to endotoxin, females ( $v s$ males) showed greater increases in depressed mood and social disconnection. Additionally, the relationships between changes in proinflammatory cytokines and social disconnection was significant for females but not for males. These findings may ultimately be useful in understanding the link between inflammation and depression, as well as sex differences in rates of depression.

\section{FUNDING AND DISCLOSURE}

This research was funded by an R01 from NIMH to NIE (5R01MH091352). We also acknowledge the additional support provided by R01AG034588; R01AG026364; R01CA160245-01; R01CA119159; R01HL095799; R01DA032922-01; P30AG028748 (to MRI); and UCLA CTSI UL1TR000124, and the Cousins Center for Psychoneuroimmunology. Additionally, the first author was supported by a predoctoral NRSA individual fellowship from NIA (1F31AG048668) and a predoctoral NRSA training fellowship from NIGMS (5T32GM084903). The aforementioned funders provided financial support for the study, but they were not involved in the conduct of the study in any other capacity (eg, design, data collection, manuscript preparation, and so on). The authors declare no conflict of interest.

\section{ACKNOWLEDGEMENTS}

We thank the staff and support of the UCLA Clinical and Translational Research Center, as well as Anthony Suffredini, MD, at the National Institutes of Health, Warren Grant Magnuson Clinical Center, for providing the standard reference endotoxin, as well as Spencer Bujarski, MA, for providing statistical consulting; neither Dr Suffredini nor Mr Bujarski were compensated.

\section{REFERENCES}

Andreasen A, Krabbe K, Krogh-Madsen R, Taudorf S, Pedersen B, Moller K (2008). Human endotoxemia as a model of systemic inflammation. Curr Med Chem 15: 1697-1705.

Baker F, Denniston M, Zabora J, Polland A, Dudley WN (2002). A POMS short form for cancer patients: psychometric and structural evaluation. Psycho-Oncology 11: 273-281.

Beck AT, Steer RA, Carbin MG (1988). Psychometric properties of the Beck Depression Inventory: twenty-five years of evaluation. Clin Psychol Rev 8: 77-100.

Bonaccorso S, Marino V, Biondi M, Grimaldi F, Ippoliti F, Maes M (2002). Depression induced by treatment with interferon-alpha in patients affected by hepatitis C virus. J Affect Disord 72: 237-241.

Breen E, Perez C, Olmstead R, Eisenberger N, Irwin M (2014). 135. Comparison of multiplex immunoassays and ELISAs for the determination of circulating levels of inflammatory cytokines. Brain Behav Immun 40: e39.

Bremmer M, Beekman A, Deeg D, Penninx B, Dik M, Hack C et al (2008). Inflammatory markers in late-life depression: results from a population-based study. J Affect Disord 106: 249-255.

Cartier A, Côté M, Lemieux I, Pérusse L, Tremblay A, Bouchard C et al (2009). Sex differences in inflammatory markers: what is the contribution of visceral adiposity? Am J Clin Nutr 89: 1307-1314.

Chapman BP, Khan A, Harper M, Stockman D, Fiscella K, Walton J et al (2009). Gender, race/ethnicity, personality, and interleukin6 in urban primary care patients. Brain Behav Immun 23: 636-642.

Covic T, Cumming SR, Pallant JF, Manolios N, Emery P, Conaghan PG et al (2012). Depression and anxiety in patients with rheumatoid arthritis: prevalence rates based on a comparison of the Depression, Anxiety and Stress Scale (DASS) and the hospital, Anxiety and Depression Scale (HADS). BMC Psychiatry 12: 6.

Cyranowski JM, Frank E, Young E, Shear MK (2000). Adolescent onset of the gender difference in lifetime rates of major depression: a theoretical model. Arch Gen Psychiatry 57: 21-27.

Duivis HE, de Jonge P, Penninx BW, Na BY, Cohen BE, Whooley MA (2011). Depressive symptoms, health behaviors, and subsequent inflammation in patients with coronary heart disease: prospective findings from the heart and soul study. Am J Psychiatry 168: 913-920.

Eisenberger NI, Inagaki TK, Mashal NM, Irwin MR (2010). Inflammation and social experience: an inflammatory challenge induces feelings of social disconnection in addition to depressed mood. Brain Behav Immun 24: 558-563. 
Eisenberger NI, Inagaki TK, Rameson LT, Mashal NM, Irwin MR (2009). An fMRI study of cytokine-induced depressed mood and social pain: the role of sex differences. NeuroImage 47: 881-890.

Epstein MM, Breen EC, Magpantay L, Detels R, Lepone L, Penugonda S et al (2013). Temporal stability of serum concentrations of cytokines and soluble receptors measured across two years in low-risk HIV-seronegative men. Cancer Epidemiol Biomarkers Prev 22: 2009-2015.

Fuller-Thomson E, Shaked Y (2009). Factors associated with depression and suicidal ideation among individuals with arthritis or rheumatism: findings from a representative community survey. Arthritis Care Res 61: 944-950.

Gimeno D, Kivimäki M, Brunner EJ, Elovainio M, De Vogli R, Steptoe A et al (2009). Associations of C-reactive protein and interleukin-6 with cognitive symptoms of depression: 12-year follow-up of the Whitehall II study. Psychol Med 39: 413-423.

Gohier B, Goeb J-L, Rannou-Dubas K, Fouchard I, Calès P, Garré J-B (2003). Hepatitis C, alpha interferon, anxiety and depression disorders: a prospective study of 71 patients. World $J$ Biol Psychiatry 4: 115-118.

Grigoriadis S, Robinson GE (2007). Gender issues in depression. Ann Clin Psychiatry 19: 247-255.

Hankin BL, Abramson LY (2001). Development of gender differences in depression: an elaborated cognitive vulnerabilitytransactional stress theory. Psychol Bull 127: 773.

Hannestad J, DellaGioia N, Ortiz N, Pittman B, Bhagwagar Z (2011). Citalopram reduces endotoxin-induced fatigue. Brain Behav Immun 25: 256-259.

Heinrich LM, Gullone E (2006). The clinical significance of loneliness: a literature review. Clin Psychol Rev 26: 695-718.

Howren MB, Lamkin DM, Suls J (2009). Associations of depression with C-reactive protein, IL-1, and IL-6: a meta-analysis. Psychosom Med 71: 171-186.

Hyde JS, Mezulis AH, Abramson LY (2008). The ABCs of depression: integrating affective, biological, and cognitive models to explain the emergence of the gender difference in depression. Psychol Rev 115: 291.

Joiner TE, Lewinsohn PM, Seeley JR (2002). The core of loneliness: Lack of pleasurable engagement-more so than painful disconnection-predicts social impairment, depression onset, and recovery from depressive disorders among adolescents. J Pers Assess 79: 472-491.

Kessler RC, McGonagle KA, Swartz M, Blazer DG, Nelson CB (1993). Sex and depression in the National Comorbidity Survey I: Lifetime prevalence, chronicity and recurrence. J Affect Disord 29: 85-96.

Koskinas J, Merkouraki P, Manesis E, Hadziyannis S (2003). Assessment of depression in patients with chronic viral hepatitis: effect of interferon treatment. Dig Dis 20: 284-288.

Kuehner C (2003). Gender differences in unipolar depression: an update of epidemiological findings and possible explanations. Acta Psychiatr Scand 108: 163-174.

Margaretten M, Julian L, Katz P, Yelin E (2011). Depression in patients with rheumatoid arthritis: description, causes and mechanisms. Int J Clin Rheumatol 6: 617-623.

McNair DM, Lorr M, Droppleman LF (1971). Manual for the Profile of Mood States. Education and Industrial Testing Service: San Diego, CA.

Nolen-Hoeksema S (2001). Gender differences in depression. Curr Direct Psychol Sci 10: 173-176.

Nolen-Hoeksema S, Girgus JS (1994). The emergence of gender differences in depression during adolescence. Psychol Bull 115: 424.
O'Connor M-F, Motivala SJ, Valladares EM, Olmstead R, Irwin MR (2007). Sex differences in monocyte expression of IL-6: role of autonomic mechanisms. Am J Physiol Regul Integr Compar Physiol 293: R145-R151.

Osborne JW, Overbay A (2004). The power of outliers (and why researchers should always check for them). Pract Assess Res Eval 9: 1-12.

Patten S, Beck C, Williams J, Barbui C, Metz L (2003). Major depression in multiple sclerosis: a population-based perspective. Neurology 61: 1524-1527.

Patten SB, Metz LM, Reimer MA (2000). Biopsychosocial correlates of lifetime major depression in a multiple sclerosis population. Mult Scler 6: 115-120.

Penninx BW, Kritchevsky SB, Yaffe K, Newman AB, Simonsick EM, Rubin $S$ et al (2003). Inflammatory markers and depressed mood in older persons: results from the Health, Aging and Body Composition study. Biol Psychiatry 54: 566-572.

Prather AA, Carroll JE, Fury JM, McDade KK, Ross D, Marsland AL (2009). Gender differences in stimulated cytokine production following acute psychological stress. Brain Behav Immun 23: 622-628.

Raison CL, Capuron L, Miller AH (2006). Cytokines sing the blues: inflammation and the pathogenesis of depression. Trends Immunol 27: 24-31.

Raison CL, Miller AH (2011). Is depression an inflammatory disorder? Curr Psychiatry Rep 13: 467-475.

Reichenberg A, Yirmiya R, Schuld A, Kraus T, Haack M, Morag A et al (2001). Cytokine-associated emotional and cognitive disturbances in humans. Arch Gen Psychiatry 58: 445-452.

Russell DW (1996). UCLA Loneliness Scale (Version 3): reliability, validity, and factor structure. J Pers Assess 66: 20-40.

Schedlowski M, Engler H, Grigoleit J-S (2014). Endotoxin-induced experimental systemic inflammation in humans: A model to disentangle immune-to-brain communication. Brain Behav Immun 35: 1-8.

Steptoe A, Hamer M, Chida Y (2007). The effects of acute psychological stress on circulating inflammatory factors in humans: a review and meta-analysis. Brain Behav Immun 21: 901-912.

Steptoe A, Owen N, Kunz-Ebrecht S, Mohamed-Ali V (2002). Inflammatory cytokines, socioeconomic status, and acute stress responsivity. Brain Behav Immun 16: 774-784.

Suffredini AF, O'Grady NP (1999). Pathophysiological responses to endotoxin in humans. In: Braude $\mathrm{H}$, Opal SM, Vogel SN, Morrison DC. (Eds.), Endotoxin in Health and Disease, First Ed. Marcel Dekker, New York, pp. 817-830.

Suffredini AF, Fantuzzi G, Badolato R, Oppenheim JJ, O'Grady NP (1999b). New insights into the biology of the acute phase response. J Clin Immunol 19: 203-214.

Van Breukelen GJ (2006). ANCOVA versus change from baseline had more power in randomized studies and more bias in nonrandomized studies. J Clin Epidemiol 59: 920-925.

Weissman MM, Bland RC, Canino GJ, Faravelli C, Greenwald S, Hwu H-G et al (1996). Cross-national epidemiology of major depression and bipolar disorder. JAMA 276: 293-299.

Weissman MM, Klerman GL (1977). Sex differences and the epidemiology of depression. Arch Gen Psychiatry 34: 98.

Whitacre CC (2001). Sex differences in autoimmune disease. Nat Immunol 2: 777-780.

Winkens B, van Breukelen GJ, Schouten HJ, Berger MP (2007). Randomized clinical trials with a pre-and a post-treatment measurement: repeated measures versus ANCOVA models. Contemp Clin Trials 28: 713-719.

Supplementary Information accompanies the paper on the Neuropsychopharmacology website (http://www.nature.com/npp) 